\title{
Bundling of Digital Goods in the Presence of Piracy
}

\author{
Chenguang (Allen) Wu \\ Hong Kong Univ. of Sci. and Tech. \\ allenwu@ust.hk
}

\author{
Chen Jin \\ National Univ. of Singapore \\ disjinc@nus.edu.sg
}

\author{
Atanu Lahiri \\ Univ. of Texas at Dallas \\ atanu.lahiri@utdallas.edu
}

\begin{abstract}
The efficacy of bundling is well-known in the context of digital goods with zero marginal cost. However, digital goods are also prone to piracy, and it is not clear what impact piracy might have on the efficacy of bundling. Prior research on this issue is limited, and it suggests that the appeal of bundling remains intact in the face of piracy. Using a model that recasts the classic bundling problem in the backdrop of piracy, we question this insight and show that piracy can severely diminish the appeal of bundling. In fact, bundling exacerbates the piracy problem and pushes more consumers to substitute the legal products with illegal ones, which more than offsets the usual benefits of bundling to a monopolist seller. Overall, the manufacturers of digital goods need to take piracy into consideration in their bundling decision and, perhaps, refrain from bundling when they anticipate the threat of piracy to be severe.
\end{abstract}

Keywords: Information goods, pricing, piracy, bundling.

\section{Introduction}

Information goods are often sold in bundles. For example, each music album is essentially a bundle of songs, and each software suite, one of multiple applications. As pointed out by Shapiro and Varian [23], bundling enables the manufacturer to extract more surplus from consumers "when it reduces the dispersion in their willingness to pay." In addition, what makes bundling particularly relevant to the case of information goods is their cost structure - as shown by Bakos and Brynjolfsson [6], the relative benefit of bundling vis-à-vis separate selling decreases with the marginal cost. Since information goods can be reproduced at a zero marginal cost, their manufacturers are expected to benefit the most from bundling.

However, aside from being zero-marginal-cost goods, information products exhibit other unique characteristics as well, and some - such as their piracy-prone nature - are highly relevant to the pricing strategy of their manufacturers. As a matter of fact, even today, piracy continues to plague various information-goods markets. A recent report by the International Federation of Phonographic Industry [14] estimates that a whopping $38 \%$ of consumers consume music illegally. Likewise, the Business Software Alliance [7] estimates that $39 \%$ of software installations are not properly licensed. In light of these facts, it is only natural that we revisit the prescription that bundling is preferable for information goods, and ask ourselves to what extent it remains applicable when the issue of piracy is accounted for. Does piracy increase or decrease the appeal of bundling? And, if indeed so, why and when?

These questions are in fact of practical significance. For example, Agarwal [2] writes, "To combat this piracy, Microsoft sells stand-alone programs like Word, PowerPoint for a lower price than the entire [Microsoft Office] suite." In a similar vein, Feldman [12] blames bundling of digital contents for increased piracy, and grumbles, "You know what's free? Illegally downloaded movies. Piracy is back. For years, consumers griped about cable bundling - having to pay high prices for hundreds of channels that they never watched in order to get the handful they did watch. The unrealized dream was that at some point cable companies would relent and offer à la carte pricing, in which customers only paid for the channels they wanted. It appears now that the streaming market saturation has led to a refracted version of this problem, show bundling. Fans don't want all of a streaming service - they only want certain shows on it." Feldman goes on to add, "So you could pay for a dozen different services to try and consume every new series and album and movie you're interested in legally... Or you could just pirate it." We seek to find out if there is any truth to such arguments by examining the interaction of piracy with bundling using a rigorous gametheoretic model.

To address our research questions, we revisit the classical bundling problem [1]. Specifically, we reexamine the conditions under which a monopolist selling two zero-marginal-cost goods would find bundling preferable to selling them separately. To 
clearly delineate the effect of piracy, we first consider the scenario in which there is no piracy. In this benchmark model, bundling predictably turns out to be the dominant strategy across the entire parameter space, echoing the result in prior research that bundling is in general preferable in the case of zeromarginal-cost goods [6].

The story changes quickly, however, when we incorporate piracy in this classical bundling problem. We do so by borrowing from prior research on piracy, where the pirated version of a product is modeled as an imperfect substitute for the original, and the level of anti-piracy enforcement determines the expected piracy cost faced by a consumer [e.g., $5,9,21]$. The resulting setup, which incorporates both bundling and piracy, is novel to the best of our knowledge, and it does lead us to some interesting insights. First, it reveals that bundling actually exacerbates piracy, and the portion of the parameter space where piracy occurs in the equilibrium considerably expands. In other words, the prevalence of piracy, to an extent, is attributable to the popularity of bundling in information-goods markets, just as claimed in the online media $[2,12]$.

Second, even though bundling makes piracy more attractive, the converse is certainly not true - piracy, in fact, makes bundling less attractive. As the manufacturer tries to raise the bundle price, piracy also increases in lockstep, seeking to erase the gains from bundling. In fact, we identify two distinct possibilities in equilibrium, one in which the benefits of bundling are strong enough to outweigh the losses from increased piracy, and another in which exactly the opposite is true. In subsequent sections, we elaborate on when the first case prevails, as well as when the other one does. In general, when piracy is more potent, for example, when the pirated product is a close substitute for the legal one, bundling is unlikely to be the dominant strategy. As the quality gap between the legal and pirated product increases, so does the appeal of bundling. These findings are instructive, as they have serious implications for any manufacturer considering bundling as a part of its pricing strategy.

\section{Literature}

The problem of bundling has been extensively studied in economics $[1,3,6]$. The main finding in this literature is that bundling decreases valuation heterogeneity among consumers, which translates to a relatively less elastic demand curve and a greater pricing power for the manufacturer. Recently, researchers have studied the applicability of this insight to novel contexts involving information goods. For example, using theoretical models, they have explored whether it holds in the presence of network effects [22]. Interestingly, it does not, and separate selling can indeed become more attractive vis-à-vis bundling. We too find a similar result in this work, albeit in a very different context involving piracy.

Moving on to the literature on piracy, it too is extensive and spans economics, marketing, and information systems. Specifically, there has been a lot of research on how a manufacturer might respond to piracy strategically, and researchers have investigated approaches such as nonlinear pricing [24], strategic patching [4], product sampling [8], strategic content delivery [16], and versioning [27]. We augment this line of research by examining the interplay of bundling with piracy.

Although the literature on piracy and bundling are both vast, there only exists limited research at their intersection. Gopal and Gupta [13] examine how a manufacturer can use bundling to combat what is known as a sharing club. The authors assume that either all products are pirated or none is, whereas, in our model, each user decides how many products to pirate and which ones to pirate. As a result, not only is our model different, but so also are our insights. For example, when the valuations and piracy costs are symmetric across products, we find that bundling can actually be dominated, a result that is in clear contrast with findings in [13]. Recently, researchers have considered the problem of bundling a software product with a cloud-based service $[28,29]$. These papers are considerably different from ours in terms of their research setup, and their insights are not comparable to ours. First, the cloud-based service happens to have a marginal cost, which becomes a significant factor in the bundling decision. Further, and more importantly, the cloudbased service cannot be pirated; it is only the software product that is prone to piracy. We place no such restrictions. Zhang et al. [29, Observation 5] claim that a low marginal cost favors bundling despite piracy, which is consistent with findings in prior literature [6]. In contrast, despite considering only zero-marginal-cost goods, we find that bundling can actually be suboptimal.

\section{Benchmark Case: No Piracy}

Let us consider the pricing problem of a monopolist that produces and sells two information goods. The question is whether the firm should sell the two products separately or sell them as a bundle, in a setting 
where each consumer strategically decides whether to use the authentic product, the pirated version, or simply neither.

We wish to first set the benchmark by investigating what happens in the absence of piracy. Then, we will bring piracy into the mix and investigate how piracy alters, if it does at all, the appeal of bundling. Both the benchmark case as well as the full setup with piracy make the following assumption:

Assumption 1. Consumers' valuation for product $i \in\{1,2\}$, denoted $v_{i}$, is uniformly distributed over $[0,1]$. Further, a consumer's valuations for the two products are independent of each other.

The uniform assumption is not critical to our findings; we simply make it to keep the model as parsimonious as possible. Regarding the assumption that a consumer's valuations for the two products are independent of each other, it is fairly common in the bundling literature [6].

As far as the costs are concerned, we make the following standard assumption [17]:

Assumption 2. The marginal cost is zero for both products, and the product development costs (fixed costs) are sunk.

When there is no piracy, each consumer must choose between buying and forgoing use. If the firm sells the two products separately, it charges the monopoly price of $\frac{1}{2}$ for each and earns a total profit of $\frac{1}{4}+\frac{1}{4}=\frac{1}{2}$. On the other hand, if it sells the two products as a bundle at price $p$, consumers would compare $v_{1}+v_{2}$ with $p$ to make their decisions. The distribution for $v_{1}+v_{2}$, therefore, determines the optimal bundle price. Accordingly, we derive the profit-maximizing price to be $\sqrt{\frac{2}{3}}$. This leads to the following benchmark.

Proposition 1. When there is no piracy in the market, bundling is the optimal strategy.

This proposition echoes the main finding in [6] that, absent a marginal cost, bundling tends to outperform individual selling. This intuition behind the result in Proposition 1 is as follows. In the absence of bundling, the demand is determined by $v_{1}$ and $v_{2}$, each of which follows an uniform distribution. However, with bundling, the distribution of interest is that of $v_{1}+v_{2}$, which happens to be triangular and more concentrated around the mean. This concentration manifests itself in the form of a lower demand elasticity and a higher pricing power for the monopolist, making bundling the preferred strategy.

\section{Model with Piracy}

We now incorporate piracy in the analysis to see how it impacts the result in Proposition 1. To do this rigorously, we borrow from prior literature, and model the pirated product as an imperfect substitute for the legal one [e.g., 4, 11, 25, 24]. There are several sources of this imperfection. First, pirated video games and software usually do not get product support from the manufacturer [18]. Second, pirated copies can contain embedded malicious codes [15]. Third, examples abound where the physical quality of the pirated product is less than that of the original product [19]. For instance, in the case of pirated movies, pirate sites often do not have access to fast content delivery networks [16] and intentionally "focus on low-resolution streaming through web sites" [26]. Finally, even when there is little perceptible difference in the physical quality between pirated and legal versions, illegally procured music or video files could be missing appropriate tags (artist, title, and genre, to name a few); the absence of tags and related indexes can make it difficult to organize and locate these files within a media player.

Assumption 3. A consumer's valuation for the pirated product is $\delta$ times his valuation for the legal one, where $\delta \in(0,1)$ is the quality degradation parameter.

Following prior research $[4,21]$, we also assume that piracy is costly to consumers and its expected value reflects the level of anti-piracy enforcement:

Assumption 4. The expected piracy cost per product is $r \geq 0$.

Following August and Tunca [4], one can view $r$ as the expected legal liability - the probability that an illegal user is successfully prosecuted times the penalty assessed on detection. In other words, $r$ depends heavily on the legal context consumers are situated in. For example, although some developing nations do not strictly enforce intellectual property laws, hundreds of piracy-related criminal prosecutions take place in the US every year under the US Federal Copyright Act (US Code Title 17), implying that $r$ could be quite significant in the US. Also, similar to the US Copyright Act, Assumption 4 treats each act of piracy as a separate infringement punishable by a separate penalty, implying that the total piracy cost is simply proportional to the number of products pirated. This is consistent with the assertion in prior literature that the piracy cost "increases monotonically with... the number of products in a product bundle" [13, p.1949]. In fact, the US Federal 
Copyright Act allows a maximum of $\$ 150,000$ per infringement as statutory damages, irrespective of the actual overall damage to the seller, and accordingly, when the Recording Industry Association of America brought hundreds of lawsuits to curb illegal sharing of music, defendants in each faced "penalties of up to $\$ 150,000$ per song" [20].

\subsection{Selling Separately in the Presence of Piracy}

If the firm charges a price $p_{i}$ for product $i$, a consumer will purchase the legal version if and only if his valuation $v_{i}$ satisfies the following individual rationality and incentive compatibility constraints.

$$
v_{i}-p_{i} \geq 0 \quad \text { and } \quad v_{i}-p_{i} \geq \delta v_{i}-r .
$$

The first condition ensures that purchasing is preferable to not using, and the second guarantees that purchasing is preferable to pirating. The resulting demand can accordingly be expressed as a function of the price:

$$
q_{i}\left(p_{i}\right)=\left(1-\max \left\{\frac{p_{i}-r}{1-\delta}, p_{i}\right\}\right) .
$$

Proposition 2. When products are sold separately, the optimal price $p_{i}^{*}, i \in\{1,2\}$, is

$$
p_{i}^{*}= \begin{cases}\frac{1-\delta+r}{2}, & \text { if } r<\frac{\delta(1-\delta)}{2-\delta}, \\ \frac{r}{\delta}, & \text { if } \frac{\delta(1-\delta)}{2-\delta} \leq r \leq \frac{\delta}{2}, \\ \frac{1}{2}, & \text { otherwise. }\end{cases}
$$

According to Proposition 2, if the products are sold separately, the profit from each is $p_{i}^{*} q_{i}\left(p_{i}^{*}\right)$. Therefore, only when bundling can generate a revenue higher than $2 p_{i}^{*} q_{i}\left(p_{i}^{*}\right)$, would the manufacturer prefer bundling. The intuition behind Proposition 2 happens to be interesting. When $r$ is extremely small, that is, anti-piracy enforcement is lacking, our monopolist tolerates piracy and sets a price of $\frac{1-\delta+r}{2}$. If $r$ is somewhat higher but not very high, the firm sets a price of $\frac{r}{\delta}$ and barely holds off the pirated product; even though there is no piracy any longer, the price, $\frac{r}{\delta}$, depends directly on $r$. This is because the threat of piracy remains in effect, and any price higher than $\frac{r}{\delta}$ allows piracy to resurface [21]. Finally, when $r$ is so high that piracy becomes completely moot, the manufacturer regains its full pricing power and switches to the usual monopoly price of $\frac{1}{2}$.
4.2. Bundling in the Presence of Piracy We now consider the case in which products are sold in a bundle. Given a bundle price $p$, a consumer will purchase the bundle only if his individual rationality constraint is satisfied, that is, $v_{1}+$ $v_{2}-p \geq 0$. Moving on to incentive compatibility, the expected surplus from pirating product $i$ is $\left(\delta v_{i}-\right.$ $r$ ). However, a consumer pirates a product only if this surplus is positive. In other words, the consumer may pirate either or both products, or pirate none. This is logical. For example, in the case of music, the consumer can just steal the song he is really interested in, and there is no obligation for him to take part in illegal sharing of other songs in the same album. Also, to steal Microsoft Word, the consumer does not have to install Microsoft Excel even though they are both a part of the same Office Suite - the consumer can simply download Microsoft Word and illegally procure a product key to activate it. So, our model is consistent with the realities of most practical situations. Of course, in special cases, such as that of sharing clubs [13], consumers may pirate all bundle constituents or pirate none. It is trivial to show that if we were to impose the same restriction - anyone pirating must pirate both products - our model would lead to results consistent with prior research that deems bundling optimal across the board. However, as we will shortly see, absent any such restriction, the dominance of bundling unexpectedly falls apart.

Since our consumer chooses whether to pirate and what to pirate, the maximum surplus he can obtain from strategically pirating is simply $\left(\delta v_{1}-r\right)^{+}+$ $\left(\delta v_{2}-r\right)^{+}$, where $x^{+}=\max \{x, 0\}$. Therefore, a consumer buys the bundle if and only if his valuations, $\left(v_{1}, v_{2}\right)$, satisfy the following:

$$
v_{1}+v_{2}-p \geq\left(\delta v_{1}-r\right)^{+}+\left(\delta v_{2}-r\right)^{+} .
$$

This condition is extremely complex and can lead to different scenarios. To compute the demand, we must first recognize that the condition in (1) results in four consumer segments:

Segment 1 (Consumers who do not consider piracy). Consider consumers satisfying $v_{1} \leq \frac{r}{\delta}$ and $v_{2} \leq \frac{r}{\delta}$. They would buy the bundle if and only if $v_{1}+v_{2} \geq p$.

Segment 2 (Consumers who might pirate either or both products). In contrast, consumers with $v_{1}>\frac{r}{\delta}$ and $v_{2}>\frac{r}{\delta}$ would buy the bundle if and only if $v_{1}+v_{2} \geq \max \left\{p, \frac{p-2 r}{1-\delta}\right\}$.

Segment 3 (Consumers who never pirate product 1 but might pirate product 2 ). Consumers satisfying $v_{1} \leq \frac{r}{\delta}$ and $v_{2}>\frac{r}{\delta}$ would purchase the bundle if and only if $v_{1}+(1-\delta) v_{2} \geq p-r$. 
Segment 4 (Consumers who never pirate product 2 but might pirate product 1 ). Finally, consider consumers with $v_{1}>\frac{r}{\delta}$ and $v_{2} \leq \frac{r}{\delta}$. They would purchase the authentic bundle if and only if $(1-\delta) v_{1}+v_{2} \geq p-r$.

Now, when $r \geq \delta$, it is obvious that the expected utility from pirating either product is at most zero. Therefore, the legal demand is simply the number of consumers for whom $v_{1}+v_{2} \geq p$. This case is similar to the one considered in Proposition 1. In other words, the issue of piracy is relevant only when $r<\delta$. Not only that, when $r<\delta$, several cases emerge as well. For each case, one has to consider each of the four segments above and then aggregate the demand from each. These cases are listed in (2).

To understand (2) clearly, we need to examine each of its cases and sub-cases separately. Since space constraints limit us from comprehensively examining them all, as an illustration, in Figure 1, we discuss only one: the sub-case $\frac{2 r}{\delta}<p \leq 1-\delta+$ $r+\frac{r}{\delta}$ of Case (ii), $\frac{\delta(1-\delta)}{2-\delta} \leq r<\delta$. In the figure, consumers located in either of the three colored regions buy the bundle. The red region (S2) represents the demand from Segment 2, the blue one (S3) from Segment 3, and the green (S4) from Segment 4. Recall that Segment 1 refers to consumers who do not consider piracy at all, Segment 2 is made up of those who consider pirating either or both, and Segments 3 and 4 comprise the ones who would consider pirating one product but not the other. In Figure 1, there is no $\mathrm{S} 1$, since there is no demand from Segment 1 in the sub-case being illustrated. The reason is that it is impossible to satisfy $v_{1} \leq \frac{r}{\delta}, v_{2} \leq \frac{r}{\delta}$, and $v_{1}+v_{2}>p$ simultaneously when $p>\frac{2 r}{\delta}$, a condition that must hold in this particular sub-case. In other words, only if the line $v_{1}+v_{2}=p$ were to be below the point $\left(\frac{r}{\delta}, \frac{r}{\delta}\right)$ in Figure 1, Segment 1 could have contributed to the bundle demand. In more general terms, for any sub-case of (2), which segments eventually contribute to the bundle demand would depend on the relative sizes of of $p, r$, and $\delta$ for that sub-case.

To elaborate on the geometry further, the conditions $p>\frac{2 r}{\delta}$ and $\frac{\delta(1-\delta)}{2-\delta} \leq r$-both necessary in this sub-case - together imply that $p>1-\delta+r$, ensuring that the point $(1, p-(1-\delta+r))$ where $(1-$ $\delta) v_{1}+v_{2}=p-r$ and $v_{1}=1$ intersect is strictly above the horizontal axis. In other words, Segment 4 is indeed a triangular region in this sub-case, exactly as depicted. A similar logic applies to Segment 3 as well. Note that the triangle in each case degenerates to a point when $p=1-\delta+r+\frac{r}{\delta}$, which is why the condition $p \leq 1-\delta+r+\frac{r}{\delta}$ is also critical to specifying the sub-case as well as its geometry. In fact, every sub-case of (2) has its own unique geometryeither in terms of the segments contributing to the bundle demand or with respect to their shapes, or possibly both.

Now, it is clear from Figure 1 that the red region has an area of $\left(1-\frac{r}{\delta}\right)^{2}-\frac{1}{2}\left(\frac{p-2 r}{1-\delta}-\frac{2 r}{\delta}\right)^{2}$. It is also clear that the green region has an area of $\frac{1}{2}\left(1-\frac{p-2 r}{1-\delta}+\frac{r}{\delta}\right)\left(\frac{r}{\delta}-p+r+1-\delta\right)$, just as does the blue one. The total demand for the sub-case follows immediately from a straightforward addition. All other sub-cases can be derived in a similar manner. The whole exercise is extremely tedious. Fortunately, however, the end result is reasonably tractable.

We are now ready to characterize the optimal pricing strategy of the manufacturer for a given $r$. Optimizing $p q(p)$, where $q(p)$ is as in (2), leads to the following important result.

Proposition 3. Let the shorthand A denote the positive square root of:

$$
\begin{array}{r}
2\left(2 \delta^{4}-4 \delta^{3} r-5 \delta^{3}+2 \delta^{2} r^{2}+2 \delta^{2} r+6 \delta^{2}+3 \delta r^{2}\right. \\
\left.+8 \delta r-5 \delta-6 r^{2}-6 r+2+\frac{3 r^{2}}{\delta}\right) .
\end{array}
$$

Then the optimal bundle price in the presence of piracy is as shown in Table 1.

The equilibrium demand can now be obtained by substituting the optimal price in Table 1 into (2), and the optimal profit, by multiplying the equilibrium demand with price. Both the optimal profit and price are non-decreasing in $r$ and non-increasing in $\delta$, which is intuitive since both a higher $r$ and a lower $\delta$ imply a weakened competition from piracy.

\subsection{Bundling vs. Separate Selling in the Presence of Piracy}

Propositions 2 and 3 together provide us the key insights that we seek in this paper. How does piracy affect the optimality of bundling? Is bundling still optimal across the board as in Proposition 1? To answer these questions, we now compare the optimal revenue from Proposition 3 with that from Proposition 2 at different $r$ and $\delta$ values. This comparison is shown in Figure 2. Note that the region above the $r=\delta$ line is uninteresting, as piracy is trivially a non-issue there. In the region below the line, which is both interesting and non-trivial, selling separately dominates in the shaded portion and bundling, in the rest of the parameter space.

Figure 2 essentially depicts the impact of piracy on the effectiveness of bundling. It shows that, in 
Case (i), $0 \leq r<\frac{\delta(1-\delta)}{2-\delta}$ :

$$
q(p)= \begin{cases}1-\frac{p^{2}}{2}, & \text { if } 0 \leq p \leq \frac{r}{\delta}, \\ \frac{1}{2}\left(\frac{2 r}{\delta}-p\right)^{2}+\left(1-\frac{r}{\delta}\right)^{2}+2\left(1-\frac{r}{\delta}\right) \frac{r}{\delta}-\left(p-\frac{r}{\delta}\right)\left(\frac{p-r}{1-\delta}-\frac{r}{\delta}\right), & \text { if } \frac{r}{\delta}<p \leq \frac{2 r}{\delta}, \\ \left(1-\frac{r}{\delta}\right)^{2}-\frac{1}{2}\left(\frac{p-2 r}{1-\delta}-\frac{2 r}{\delta}\right)^{2}+\left(2-\frac{2 p-3 r}{1-\delta}+\frac{r}{\delta}\right) \frac{r}{\delta}, & \text { if } \frac{2 r}{\delta}<p \leq 1-\delta+r, \\ \left(1-\frac{r}{\delta}\right)^{2}-\frac{1}{2}\left(\frac{p-2 r}{1-\delta}-\frac{2 r}{\delta}\right)^{2}+\left(1-\frac{p-2 r}{1-\delta}+\frac{r}{\delta}\right)\left(\frac{r}{\delta}-p+r+1-\delta\right), & \text { if } 1-\delta+r<p \leq 1-\delta+r+\frac{r}{\delta}, \\ \frac{1}{2}\left(\frac{p-2 r}{1-\delta}-2\right)^{2}, & \text { if } 1-\delta+\frac{r}{\delta}<p \leq 2(1-\delta+r) .\end{cases}
$$

Case (ii), $\frac{\delta(1-\delta)}{2-\delta} \leq r<\delta$ :

$$
q(p)= \begin{cases}1-\frac{p^{2}}{2}, & \text { if } 0 \leq p \leq \frac{r}{\delta}, \\ \frac{1}{2}\left(\frac{2 r}{\delta}-p\right)^{2}+\left(1-\frac{r}{\delta}\right)^{2}+2\left(1-\frac{r}{\delta}\right) \frac{r}{\delta}-\left(p-\frac{r}{\delta}\right)\left(\frac{p-r}{1-\delta}-\frac{r}{\delta}\right), & \text { if } \frac{r}{\delta}<p \leq 1-\delta+r, \\ \frac{1}{2}\left(\frac{2 r}{\delta}-p\right)^{2}+\left(1-\frac{r}{\delta}\right)^{2}+\left(\frac{3 r}{\delta}-2 p+r+1-\delta\right)\left(1-\frac{r}{\delta}\right), & \text { if } 1-\delta+r<p \leq \frac{2 r}{\delta}, \\ \left(1-\frac{r}{\delta}\right)^{2}-\frac{1}{2}\left(\frac{p-2 r}{1-\delta}-\frac{2 r}{\delta}\right)^{2}+\left(1-\frac{p-2 r}{1-\delta}+\frac{r}{\delta}\right)\left(\frac{r}{\delta}-p+r+1-\delta\right), & \text { if } \frac{2 r}{\delta}<p \leq 1-\delta+r+\frac{r}{\delta}, \\ \frac{1}{2}\left(\frac{p-2 r}{1-\delta}-2\right)^{2}, & \text { if } 1-\delta+r+\frac{r}{\delta}<p \leq 2(1-\delta+r) .\end{cases}
$$

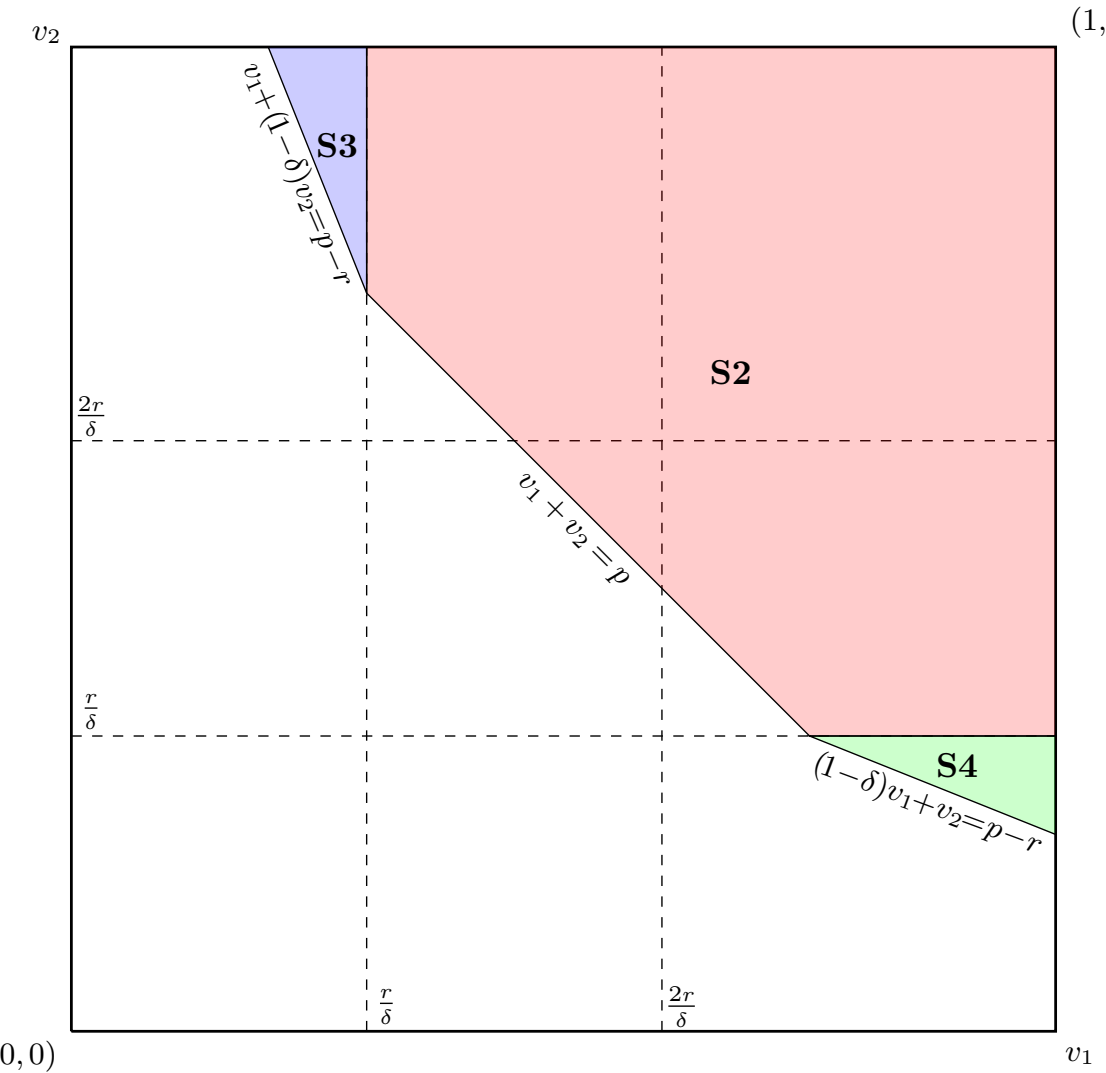

$(0,0)$

Figure 1. Bundle Demand in the Presence of Piracy When $\frac{\delta(1-\delta)}{2-\delta} \leq r<\delta$ and $\frac{2 r}{\delta}<p \leq 1-\delta+r+\frac{r}{\delta}$; S2-S4 Represent Demands from Segments 2-4; Demand from Segment 1 is 0 in This Case 
Table 1. Optimal Bundle Price with Piracy

\begin{tabular}{|c|c|c|}
\hline Range for $\delta$ & $p^{*}$ & Range for $r$ \\
\hline$\delta \in\left(0, \frac{2}{3}\right]$ & $\begin{array}{c}\frac{4 r+\sqrt{2\left(3 \delta^{2}-6 \delta+5 r^{2}+3-\frac{3 r^{2}}{\delta}\right)}}{3} \\
\frac{4 r+\sqrt{2\left(3-\frac{3 r^{2}}{\delta}+5 r^{2}-3 \delta^{2}\right)}}{3(1+\delta)} \\
\sqrt{\frac{2}{3}}\end{array}$ & $\begin{array}{l}\text { if } 0 \leq r \leq \delta \sqrt{\frac{1-\delta}{6-\delta}} \\
\text { if } \delta \sqrt{\frac{1-\delta}{6-\delta}}<r<\delta \sqrt{\frac{2}{3}} \\
\text { if } r \geq \delta \sqrt{\frac{2}{3}}\end{array}$ \\
\hline$\delta \in\left(\frac{2}{3}, 1\right)$ & $\begin{array}{c}\frac{4 r+\sqrt{2\left(3 \delta^{2}-6 \delta+5 r^{2}+3-\frac{3 r^{2}}{\delta}\right)}}{3} \\
\frac{\frac{4 \delta r+8 \delta-4-4 \delta^{2}+A}{3(2 \delta-1)}}{3-\sqrt{2\left(3 \delta-6 r+2+\frac{3 r^{2}}{\delta}\right)}} \\
\frac{4 r+\sqrt{2\left(3-\frac{3 r^{2}}{\delta}+5 r^{2}-3 \delta^{2}\right)}}{3(1+\delta)} \\
\sqrt{\frac{2}{3}}\end{array}$ & $\begin{array}{l}\text { if } 0 \leq r \leq \frac{\delta(1-\delta)}{3 \delta-2}\left(\sqrt{4-\frac{2}{\delta}}-1\right) \\
\text { if } \frac{\delta(1-\delta)}{3 \delta-2}\left(\sqrt{4-\frac{2}{\delta}}-1\right)<r<\frac{\delta(2-\delta)}{6-\delta} \\
\text { if } \frac{\delta(2-\delta)}{6-\delta} \leq r<\frac{\delta}{3 \delta-2}\left(3 \delta-1-\sqrt{2\left(2-\frac{1}{\delta}\right)}\right) \\
\text { if } \frac{\delta}{3 \delta-2}\left(3 \delta-1-\sqrt{2\left(2-\frac{1}{\delta}\right)}\right) \leq r<\delta \sqrt{\frac{2}{3}} \\
\text { if } r \geq \delta \sqrt{\frac{2}{3}}\end{array}$ \\
\hline
\end{tabular}

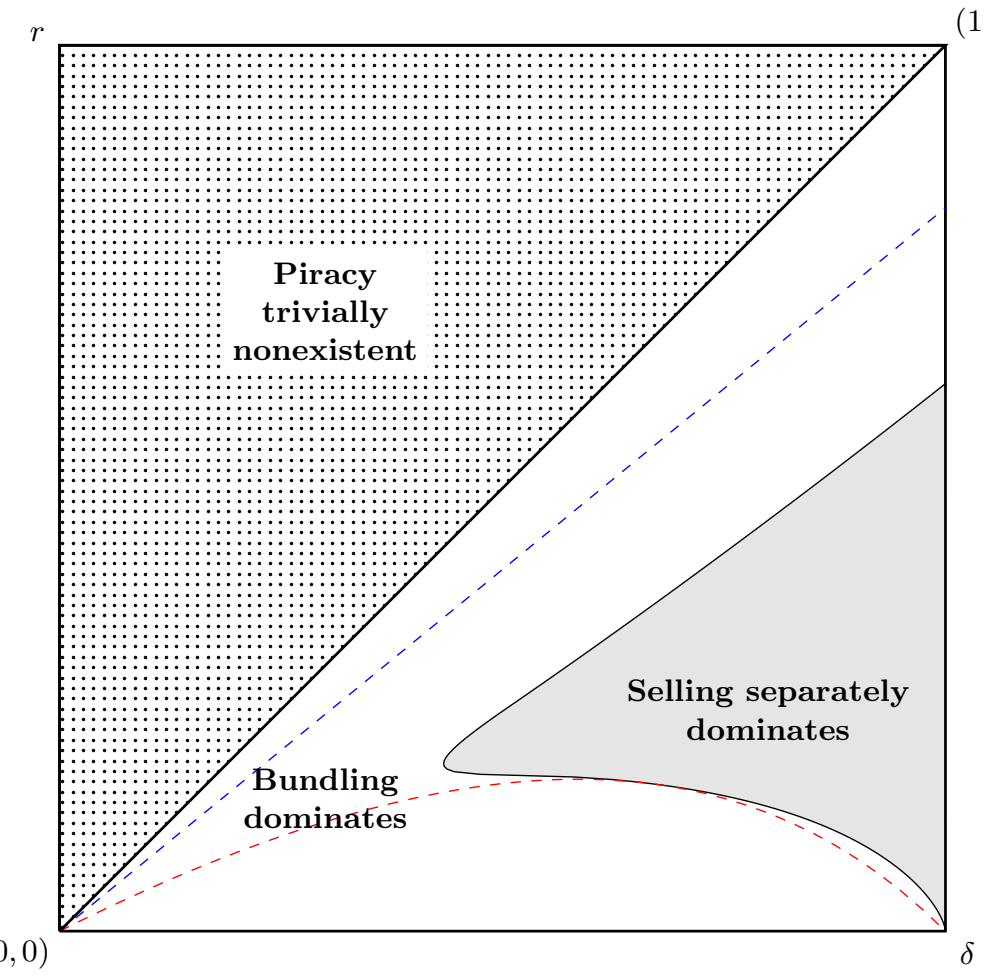

Figure 2. Bundling vs. Separate Selling in the Presence of Piracy

the presence of piracy, bundling is no longer optimal across the board. More importantly, the shaded region is sizable, naturally making it both significant and relevant from a practical standpoint. Furthermore, as $\delta$ increases, so does the range of values of $r$ for which selling separately is now optimal. This is reflected in the funnel-like shape of the shaded region. The funnel shape is in fact instructive. Since, in many real-world contexts, pirated products are reasonably close substitutes for the legal one - that is, $\delta$ is often close to 1 in reality - piracy ought to merit a serious consideration from digital-goods 
manufacturers seeking to use bundling. Instead of presuming that bundling is automatically optimal for zero-marginal-cost goods, manufacturers should carefully evaluate all its options.

What explains such an impact of piracy on the optimality of bundling? In particular, why is bundling no longer the best strategy across the board? To understand, let us first note that, in situations where $\delta$ is sufficiently large, a very high $r$ or a very low $r$ works in favor of bundling, whereas quite the opposite happens in the region where $r$ is moderate. In other words, there are two boundaries that separates the regions where bundling is optimal and where it is not: (i) the bottom boundary where we cross over from a low $r$ to a moderate one and (ii) the top boundary where we cross over from a moderate $r$ to a high one. The rationales behind these two boundaries are somewhat different. The top boundary is nearly linear and can be approximated by an upward sloping line. In other words, this boundary implies that if $r$ is larger than a certain multiple of $\delta$, bundling takes over as the dominant strategy. This is because when $r$ is significantly large compared to $\delta$, piracy is not potent enough to make any material difference to the manufacturer's strategy, and we are essentially back to the world where bundling is known to prevail.

Now, the lower boundary is a bit different. It arises from the fact that, at a moderate $r$, selling separately is quite effective in mitigating piracy whereas bundling is not. A closer look at the two dashed lines in Figure 2 makes this point quite apparent. The red-dashed line represents the threshold above which selling separately becomes effective in holding piracy to a nominal level, and the blue-dashed line represents the point where bundling is able to achieve the same. ${ }^{1}$ Clearly, between the two lines, separate selling is more effective in controlling piracy, which is also why it can also dominate bundling profit-wise in a good part of the region in-between. Below the red dashed line, however, neither strategy is effective in controlling piracy, so the usual dominance of bundling remains intact.

The broader takeaway is apparent. Piracy makes bundling less attractive only because bundling makes piracy more attractive. To the best of our

\footnotetext{
${ }^{1}$ When the manufacturer sells separately, piracy occurs in the equilibrium only in the region below the red curve; see Proposition 2 and the adjoining discussion. However, when the manufacturer resorts to bundling, the piracy region expands considerably and swallows the entire space below the blue straight line. As apparent from Proposition 3, the equation of this line is $r=\delta \sqrt{\frac{2}{3}}$.
}

knowledge, this is a new insight, and it augments the existing literature on bundling and piracy. More importantly, it is also practically relevant. As discussed in the introduction, bundling has already been blamed for higher piracy rates in certain markets $[2,12]$. So, the possibility that piracy could be having a significant negative effect on the usefulness of bundling should not be ignored by sellers in those markets.

\section{Conclusion}

Bundling is quite popular in various informationgoods markets. This popularity owes itself, in part, to the underlying cost structure - as shown in prior research, bundling is particularly preferable to separate selling when the bundle constituents can be produced at a zero marginal cost [6]. Thanks to its solid microeconomic foundations, this wisdom has long become the primary yardstick for assessing the efficacy of bundling in many information-goods markets. In this work, we put this wisdom under spotlight once again, in order to test its generalizablity to contexts afflicted with piracy.

Our endeavor is of obvious practical importance. Information goods are not only unique with respect to their cost structure, but they are also so when it comes to their easy reproducibility, a characteristic that naturally makes them vulnerable to piracy. Given how widespread piracy is today and how broad its reach has become, it is indeed important to find out whether the well-known efficacy of bundling for zero-marginal-cost goods would also hold in a setting fraught with piracy. To this end, we employ a parsimonious setup that involves two important parameters - $\delta$, which captures the quality difference between the legal and illegal products, and $r$ that captures the cost of piracy. The interesting question is then: Is the superiority of bundling anyway contingent on $r$ and $\delta$ ?

The answer to the above question happens to be a resounding 'yes.' Figure 2, which compares the optimal profit obtained from Proposition 3 with that from Proposition 2, makes this amply clear. As shown in that figure, there is a sizable region in the $(\delta, r)$ space where bundling is no longer optimal despite the assumption of a zero marginal cost. This region, where bundling is now dominated, has the shape of a funnel - it is the widest near $\delta=1$, and as $\delta$ becomes smaller, it gradually tapers off, implying that the range of values of $r$ for which piracy renders bundling ineffective is non-decreasing in $\delta$.

The economic underpinning of Proposition 3 is quite interesting. The primary benefit of bundling is 
that it reduces demand elasticity and increases the pricing power of the monopolist. However, raising the price is possible only if the monopolist is still in command of its monopoly power, which is clearly not the case at large values of $\delta$. In fact, when the illegal product is a very close substitute for the legal one, piracy offers the manufacturer a very strong competition. The net result is a significant reduction in the monopolist's pricing power and a sharp decline in its ability to profit from bundling.

The overall implication is clear. Bundling of information goods is far less useful in contexts fraught with piracy. When the pirated product is close to the legal one in terms of quality, a zero marginal cost does not automatically make bundling the dominant strategy. The manufacturer must proceed with abundant caution and properly evaluate both optionsbundling and separate selling-with appropriate market research, instead of rushing to conclusions.

\section{Ongoing Research}

How widely applicable is the insight that piracy makes bundling less appealing to a manufacturer? Does it apply to situations where bundling has additional advantages vis-à-vis separate selling? To answer these questions, we are now examining the applicability of our findings to various practical situations. For example, it is important to find out what happens when consumers' valuations for the two bundle constituents are negatively correlated. In general, a strong negative correlation is expected to favor bundling [1]. So, it is not immediately clear if our results would extend to settings with such correlations. Also, how piracy impacts the efficacy of mixed bundling remains an open question. Mixed bundling, in general, is superior to bundling, as it entails a better segmentation of the consumer market. So, it is important to verify if our insights with respect to the impacts of piracy on pure bundling would carry over to mixed bundling. Our preliminary investigation suggests that they indeed do, although only qualitatively.

There are a few other issues to consider as well, for example, the possibility of an endogenous piracy cost, which is particularly likely if manufacturers are able to lobby with the government for stricter anti-piracy enforcement [21]. There is also an obvious need to verify that our results indeed extend to settings with more than two products. We are currently in the process of rigorously analyzing all these possibilities, in order to develop a more comprehensive picture of the interplay between piracy and bundling.
Finally, we assumed a monopoly setting, and it is not clear whether the insights from our work would carry over to a scenario where competition is significant. Broadly speaking, the monopoly assumption has merits. Information goods, by their very nature, affords their manufacturers some monopoly power-there is only one software like Microsoft Word, only one television series like The Game of Thrones, and only one movie like Life is Beautiful. The examples mentioned in the introduction of this work also point to consumers' disenchantment with high prices, which again is reflective of substantial monopoly power. Naturally, the presumption of a monopoly or near-monopoly is not only realistic in our context, but it is also quite common in the existing literature on bundling of information goods [e.g., $6,22]$. Since our primary objective is to see whether the insights from this literature actually hold in the presence of piracy, we employ a similar monopoly setup; doing so facilitates an apple-to-apple comparison of our results to the ones in prior works, which in turn makes it easier for us to isolate the impact of piracy on the usefulness of bundling.

Nevertheless, the issue of competition could be important for certain manufacturers. While we leave the task of analyzing competition to future research, it would be remiss of us to not note that piracy essentially plays the role of a shadow competitor. In our setup, piracy injects competition into an otherwise monopolistic market, making it difficult for the manufacturer to enjoy its monopoly power that is critical to reaping the full benefit of bundling. Since competition among manufacturers also dents their pricing power, it would seem that competition itself will keep prices somewhat low, and piracy under control, making the latter less relevant in the process. Further, stronger competition among manufacturers might add to lure of bundling [10], making our results somewhat less critical in competitive settings. Additional research is necessary to developing a clear intuition about these interactions.

\section{References}

[1]W. Adam and J. Yellen, "Commodity Bundling and the Burden of Monopoly," Quarterly Journal of Economics, 1976 (90:3), pp. 475-498.

[2]P. Agarwal, "Microsoft's Bundling Policy," Intelligent Economist, March 2017.

[3]M. Armstrong, "Price Discrimination by a ManyProduct Firm," Review of Economic Studies, 1999 (66:1), pp. 151-168. 
[4]T. August and T. Tunca, "Let the Pirates Patch? An Economic Analysis of Network Software Security Patch Restrictions," Information Systems Research, 2008 (19:1), pp. 48-70.

[5]S.-H. Bae and J. Choi, "A Model of Piracy," Information Economics and Policy, 2006 (18:3), pp. 303-320.

[6]Y. Bakos and E. Brynjolfsson, "Bundling and Competition on the Internet," Marketing Science, 2000 (19:1), pp. 63-82.

[7]BSA, "Global Software Survey," Business Software Alliance, May 2016.

[8]R. Chellappa and S. Shivendu, "Managing Digital Piracy: Pricing and Sampling Strategies for Digital Experience Goods in Vertically Segmented Markets," Inform. Systems Res., 2005 (16:4), pp. 400-417.

[9]Y.-N. Chen and I. Png, "Information goods pricing and copyright enforcement: Welfare analysis," Information Systems Research, 2003 (14:1), pp. 107-123.

[10]H.-L. Chung, Y.-S. Lin, and J.-L. Hu, "Bundling strategy and product differentiation," Journal of Economics, 2013 (108:3), pp. 207-229.

[11]D. Dey, A. Kim, and A. Lahiri, "Online Piracy and the "Longer Arm" of Enforcement," Management Science, 2019 (65:3), pp. 1173-1190.

[12]B. Feldman, "Piracy is back," Intelligencer, June 2019.

[13]R. Gopal and A. Gupta, "Trading higher software piracy for higher profits: The case of phantom piracy," Management Science, 2010 (56:11), pp. 1946-1962.

[14]IFPI, "Music Consumer Insight Report," International Federation of Phonographic Industry, October 2018 .

[15]J. Jaisingh, "Impact of piracy on innovation at software firms and implications for piracy policy," Decision Support Systems, 2009 (46:4), pp. 763-773.

[16]M. Johar, N. Kumar, and V. Mookerjee, "Content Provision Strategies in the Presence of Content Piracy," Information Systems Research, 2012 (23:3), pp. 960-975.

[17]R. Jones and H. Mendelson, "Information Goods vs. Industrial Goods: Cost Structure and Competition," Management Science, 2011 (57:1), pp. 164-176.

[18]K. Kannan, M. Rahman, and M. Tawarmalani, "Economic and Policy Implications of Restricted Patch Distribution," Management Science, 2016 (62:11), pp. 3161-3182.

[19]J. Karaganis, "Rethinking Piracy," in Media Piracy in Emerging Economies, J. Karaganis, ed., Social Science Research Council, 2011, pp. 1-73.
[20]P. R. La Monica, "Music industry sues swappers," CNN Money, September 2003.

[21]A. Lahiri and D. Dey, "Effects of Piracy on Quality of Information Goods," Management Science, 2013 (59:1), pp. 245-264.

[22]A. Prasad, R. Venkatesh, and V. Mahajan, "Optimal Bundling of Technological Products with Network Externality," Management Science, 2018 (56:12), pp. 2224-2236.

[23]C. Shapiro and H. Varian, Information Rules: A Strategic Guide to the Network Economy, Boston: Harvard Business School Press, 1999.

[24]A. Sundararajan, "Managing Digital Piracy: Pricing and Protection," Information Systems Research, 2004 (15:3), pp. 287-308.

[25]T. Tunca and Q. Wu, "Fighting Fire with Fire: Commercial Piracy and the Role of File Sharing on Copyright Protection Policy for Digital Goods," Information Systems Research, 2013 (24:2), pp. 436-453.

[26]A. Wohl, "A new paradigm for dealing with illegal redistribution of content," Cisco Blogs, October 2016.

[27]S.-Y. Wu and P.-Y. Chen, "Versioning and Piracy Control for Digital Information Goods," Operations Research, 2008 (56:1), pp. 157-172.

[28]X. Zhang and W. Yue, "Bundling of Software Produces and Services to Fight Against Piracy," in Proceedings of the Thirty-Fourth International Conference on Information Systems, 2013.

[29]X. Zhang, W. Yue, and W. Hui, "Software piracy and bundling in the cloud-based software era," Information Technology \& People, 2019. 